\title{
Invariance groups of functions and related Galois connections
}

\section{Eszter K. Horváth ${ }^{1}$ (D) $\cdot$ Reinhard Pöschel $^{2}$ (D) $\cdot$ Sven Reichard ${ }^{3}$}

Received: 28 February 2020 / Accepted: 3 August 2020 / Published online: 23 October 2020

(c) The Author(s) 2020, corrected publication 2020

\begin{abstract}
Invariance groups of sets of Boolean functions can be characterized as Galois closures of a suitable Galois connection. We consider such groups in a much more general context using group actions of an abstract group and arbitrary functions instead of Boolean ones. We characterize the Galois closures for both sides of the corresponding Galois connection and apply the results to known group actions.
\end{abstract}

Keywords Invariance group - Group action - Galois connection · Boolean function · Permutation group

Mathematics Subject Classification 20B25 $\cdot$ 20B35 $\cdot$ 06A15 $\cdot 05 \mathrm{E} 18$

\section{Introduction}

An $n$-ary Boolean function $f$ is invariant under a permutation $\sigma \in S_{n}$ (notation $\sigma \vdash f$ ) iff permuting the variables of $f$ according to $\sigma$ does not change the function value: $f\left(x_{\sigma(1)}, \ldots, x_{\sigma(n)}\right)=f\left(x_{1}, \ldots, x_{n}\right)$. The invariance group of $f$ consists of all permutations $\sigma$ with $\sigma \vdash f$.

This research of the first author was supported by NFSR of Hungary (OTKA), grant number K 115518 , and by grant TUDFO/47138-1/2019-ITM of the Ministry for Innovation and Technology, Hungary.

$凶 \quad$ Reinhard Pöschel

Reinhard.Poeschel@tu-dresden.de

Eszter K. Horváth

horeszt@math.u-szeged.hu

http://www.math.u-szeged.hu/ horvath

Sven Reichard

Sven.Reichard@tu-dresden.de

1 Bolyai Institute, University of Szeged, Szeged, Hungary

2 Technische Universität Dresden, Dresden, Germany

3 Dresden International University, Dresden, Germany 
Invariance groups of Boolean functions are important objects of study in computer science (see Clote and Kranakis (1991) and the references therein). Algebraic investigations were started by Kisielewicz (1998) who called such groups symmetry groups. In Kisielewicz (1998) also $k$-valued Boolean functions and their symmetry groups are considered, which coincides with invariance groups of sets of ordinary Boolean functions (just taking the intersection of all invariance groups of functions in the given set). This led to the question how one can determine or characterize the invariance groups of sets of Boolean functions.

There are several contributions to this problem, e.g., Grech (2010), Grech and Kisielewicz (2014) (giving a full answer for subgroups of direct sums of regular permutation groups), Horváth et al. (2015) (considering a finer classification using functions on a $k$-element set instead of Boolean functions), Xiao (2005) (generalization by considering the linear group $\operatorname{GL}(n, 2)$ instead of $\left.S_{n}\right)$.

The above relation $\vdash$ induces a Galois connection between permutations and Boolean functions, such that the invariance groups (of sets of Boolean functions) are just the Galois closed sets of permutations.

In the present paper we consider this Galois connection in a much more general context, namely instead of Boolean functions $f \in 2^{2^{n}}$ (throughout the paper 2 mostly will stand for the two-element set $\{0,1\}$ ) we consider arbitrary functions $f \in K^{B}$, and instead of the action of $S_{n}$ on the $n$-tuples $\left(x_{1}, \ldots, x_{n}\right) \in 2^{n}$ we consider the action of an arbitrary (abstract) group $\Gamma$ on the set $B$. Specializing this to $\Gamma=S_{n}$ and $B=2^{n}$ we return to the "classical" case of permutations and Boolean functions. Moreover, we characterize not only the invariance groups, i.e., the Galois closures on the group side, but we also characterize the Galois closures on the other side of the Galois connection, i.e., on the function side. In the "classical" case this means the following: starting with some set $F$ of Boolean functions, we can characterize how to construct from them all Boolean functions invariant under all permutations from the invariance group of $F$.

All this can be realized on the background of two helpful transformations of the "language", namely, the functions $f \in K^{B}$ can be reduced to functions $f \in 2^{B}$ (without changing the invariance groups, cf. 3.7) and then $2^{B}$ will be interpreted as the power set $\mathfrak{P}(B)$ (cf. 3.8). For Boolean functions even a further change - from $B=2^{n}$ to $\mathfrak{P}([n])$ (cf. Sect. 5) - comes into play, i.e., instead of Boolean functions there can be considered sets of subsets of $[n]$, which are just hypergraphs and are called unordered relations in Dalla Volta and Siemons (2009), also mentioned in Grech and Kisielewicz (2014). A further helpful tool for our results is the well-known Galois connection Aut - Inv (this is the restriction (to permutations) of Pol - Inv, the "most basic Galois connection in algebra", as emphasized in McKenzie et al. (1987, p. 147), whose Galois closures are well understood. We want to mention here also the work of Woldar (2020) who considers geometric groups of second order (i.e., automorphism groups of sets of hypergraphs). This fits into our approach by setting $\Gamma=S_{n}$ acting on $B=2^{2^{n}}$.

The paper is organized as follows. In Sect. 2 we introduce needed notions and notations for group actions and Galois connections in general, in particular also the above mentioned "most basic Galois connection". In Sect. 3 the Galois connection of our main interest (induced by $\vdash$ ) is considered in its most general form and the Galois closures, in particular the invariance groups, are characterized (Theorems 3.5, 
3.6 for permutations, and Theorem 3.9 for functions $f \in 2^{B}$, equivalently for sets $\mathfrak{U} \subseteq \mathfrak{P}(B)$ of subsets of $B)$. In the further sections we apply the results of Sect. 3 to concrete group actions: Sect. 4 deals with the "trivial" case of the natural action of a permutation group $\left(\Gamma=S_{n}, B=[n]\right)$, in Sect. 5 we consider the "classical" case of Boolean functions $\left(\Gamma=S_{n}, B=2^{n}\right.$ or $\left.B=\mathfrak{P}([n])\right)$ and in Sect. 6 we take the linear group $\Gamma=\operatorname{GL}(n, 2)$ acting on the vector space $V:=B:=2^{n}$.

Since our general Theorem 3.6 (construction of invariance groups) was a generalization of known results for the actions in Sects. 5 and 6, its application to these special cases gives (in principle) nothing new. The Galois closures on the other side of the Galois connection here might be of more interest, since for them we had to provide a characterization of the groups $\widehat{S}_{n}\left(S_{n}\right.$ acting on $\left.B=\mathfrak{P}([n])\right)$ and $L_{n}=\operatorname{GL}(n, 2)$ (acting on $B=V=2^{n}$ ) by invariant relations on the set $B$ (Propositions 5.1, 6.1). Moreover, for invariance groups of Boolean functions (Sect. 5) a complete characterization via invariant relations (i.e., as Galois closures of the Galois connection Aut - Inv) can be given (Theorem 5.3).

Some examples in Sects. 5 and 6 illustrate our results, in particular we also give the total number of invariance groups for small $n$.

\section{Preliminaries}

Actions 2.1 Let $\Gamma=(\Gamma, \cdot, \varepsilon)$ be a (finite) group (with identity element $\varepsilon$ ). A group action of $\Gamma$ on a set $B$, notation $\Gamma \curvearrowright B$, is a mapping $B \times \Gamma \rightarrow B:(b, \sigma) \mapsto b^{\sigma}$ satisfying $x^{\varepsilon}=x$ and $\left(x^{\sigma}\right)^{\tau}=x^{\sigma \tau}$ for all $x \in B$ and $\sigma, \tau \in \Gamma$. Equivalently, a group action $\Gamma \curvearrowright B$ is determined by a permutation representation, i.e., a group homomorphism $h: \Gamma \rightarrow \operatorname{Sym}(B)$ (Sym denotes the full symmetric group on $B$ ) where $h(\sigma)$ is the permutation defined by $h(\sigma): b \mapsto b^{\sigma}$. If necessary we write $\Gamma \curvearrowright_{h} B$. The image of $h$ is a subgroup of $\operatorname{Sym}(B)$ and will be denoted by $\widehat{\Gamma}$ if the corresponding action is fixed and we also write $\widehat{\sigma}$ for $h(\sigma)$. The natural action of a permutation $\pi \in \operatorname{Sym}(B)$ on $B$ is also denoted by $b^{\pi}:=\pi(b)$. Thus we have $b^{\sigma}=b^{\widehat{\sigma}}$ for any $\sigma \in \Gamma, b \in B$.

For a subgroup $G \leq \Gamma$ let

$$
\operatorname{Orb}_{B} G:=\left\{b^{G} \mid b \in B\right\}, \text { where } b^{G}:=\left\{b^{\sigma} \mid \sigma \in G\right\},
$$

denote the set of all orbits of $G$ (under the group action). For $b \in B$ and $C \subseteq B$ let

$\Gamma_{b}:=\left\{\sigma \in \Gamma \mid b^{\sigma}=b\right\}$ and $\Gamma_{C}:=\left\{\sigma \in \Gamma \mid C^{\sigma}=C\right\}$, where $C^{\sigma}:=\left\{b^{\sigma} \mid b \in C\right\}$,

be the corresponding (point and set) stabilizers.

Galois connections 2.2 Let $I \subseteq M_{1} \times M_{2}$ be a binary relation between sets $M_{1}$ and $M_{2}$. The Galois connection induced by $I$ is the pair $(\varphi, \psi)$ of the following mappings:

$$
\begin{aligned}
\varphi: \mathfrak{P}\left(M_{1}\right) \rightarrow \mathfrak{P}\left(M_{2}\right): X \mapsto X^{I}, & \psi: \mathfrak{P}\left(M_{2}\right) \rightarrow \mathfrak{P}\left(M_{1}\right): Y \mapsto Y^{I}, \\
\text { where } X^{I}:=\left\{y \in M_{2} \mid \forall x \in X: x I y\right\}, & Y^{I}:=\left\{x \in M_{1} \mid \forall y \in Y: x I y\right\} .
\end{aligned}
$$


A pair $(\varphi, \psi)$ of mappings as above is a Galois connection (for suitable $I$ ) if and only if it satisfies

$$
\forall X \subseteq M_{1}, Y \subseteq M_{2}: X \subseteq Y^{I} \Longleftrightarrow X^{I} \supseteq Y
$$

For $X \subseteq M_{1}$, the set $X^{I I}$ is called the Galois closure of $X$, anlogously $Y^{I I}$ is the Galois closure of $Y \subseteq M_{2}$; if $X^{I I}=X$ or $Y^{I I}=Y$ then these sets are called Galois closed. The mappings $X \mapsto X^{I I}$ and $Y \mapsto Y^{I I}$ are closure operators. The main problem for each Galois connection is the characterization of its Galois closures (it easily can be seen that they are just the sets of the form $Y^{I}$ and $X^{I}$ ).

For particular Galois connection usually special notations are introduced. We give a "classical" example which will be needed later.

Example 2.3 (The Galois connections Pol - Inv and Aut - Inv)

Let $\operatorname{Op}^{(n)}(A)\left(\operatorname{Rel}^{(m)}(A)\right)$ denote the set of all $n$-ary operations $f: A^{n} \rightarrow A(m$ ary relations $\left.\varrho \subseteq A^{m}\right)$ on a set $A$ and $\operatorname{Op}(A):=\bigcup_{n \in \mathbb{N}_{+}} \operatorname{Op}^{(n)}(A)(\operatorname{Rel}(A):=$ $\bigcup_{m \in \mathbb{N}_{+}} \operatorname{Rel}^{(m)}(A)$, respectively). A function $f: A^{n} \rightarrow A$ preserves a relation $\varrho \subseteq A^{m}$ (or $f$ is a polymorphism of $\varrho$, or $\varrho$ is invariant for $f$ ) if $r_{1}, \ldots, r_{n} \in \varrho$ implies $f\left[r_{1}, \ldots, r_{n}\right] \in \varrho$ for all $r_{i}=\left(r_{i 1}, \ldots, r_{i m}\right)(i \in\{1, \ldots, n\})$ where

$$
f\left[r_{1}, \ldots, r_{n}\right]:=\left(f\left(r_{11}, \ldots, r_{n 1}\right), \ldots, f\left(r_{1 m}, \ldots, r_{n m}\right)\right)
$$

is defined componentwise. This preservation property is denoted by $f \triangleright \varrho$. If we take this relation $\triangleright$ for $I$, then, according to 2.2, we get a Galois connection between $M_{1}=\mathrm{Op}(A)$ and $M_{2}=\operatorname{Rel}(A)$. The corresponding operators for $F \subseteq \mathrm{Op}(A)$ and $Q \subseteq \operatorname{Rel}(A)$ are denoted as follows:

$$
\begin{aligned}
& \text { Pol } Q:=Q^{\triangleright}=\{f \in \mathrm{Op}(A) \mid \forall \varrho \in Q: f \triangleright \varrho\}(\text { polymorphisms of } Q), \\
& \text { Inv } F:=F^{\triangleright}=\{\varrho \in \operatorname{Rel}(A) \mid \forall f \in F: f \triangleright \varrho\}(\text { invariants of } F) .
\end{aligned}
$$

Restricting this Galois connection to permutations (i.e., taking $M_{1}:=\operatorname{Sym}(A)$ ), we get the Galois connection Aut - Inv:

$$
\begin{aligned}
& \text { Aut } Q:=\{\sigma \in \operatorname{Sym}(A) \mid \forall \varrho \in Q: \sigma \triangleright \varrho\}(\text { automorphisms of } Q \subseteq \operatorname{Rel}(A)), \\
& \text { Inv } G:=\{\varrho \in \operatorname{Rel}(A) \mid \forall \sigma \in G: \sigma \triangleright \varrho\} \text { (invariants of } G \subseteq \operatorname{Sym}(A)) .
\end{aligned}
$$

Note that $\sigma \triangleright \varrho\left(\right.$ for $\left.\sigma \in \operatorname{Sym}(A), \varrho \in \operatorname{Rel}^{(m)}(A)\right)$ simply means $\left(a_{1}^{\sigma}, \ldots, a_{m}^{\sigma}\right) \in \varrho$ for every $\left(a_{1}, \ldots, a_{m}\right) \in \varrho$.

Sometimes we write $\operatorname{Aut}_{A} Q$ and $\operatorname{Inv}_{A} G$ if we want to mention the base set $A$ explicitly. Moreover, for $m \in \mathbb{N}_{+}$, we put $Q^{(m)}:=Q \cap \operatorname{Rel}^{(m)}(A)$ and $\operatorname{Inv}^{(m)} F:=$ $(\operatorname{Inv} F)^{(m)}$.

The Galois closures are known and can be characterized as follows. We assume from now on that $A$ is finite (to avoid some modifications which would be necessary otherwise). Then, on the functional side, we get exactly the clones [i.e., sets of operations closed under composition and containing all projections, see, e.g., Kerkhoff et al. 
(2014)] and the permutation groups as Galois closures, i.e., $\langle F\rangle_{\mathrm{Op}(A)}=\operatorname{Pol} \operatorname{Inv} F$ is the clone generated by $F \subseteq \operatorname{Op}(A)$, and $\langle G\rangle_{\operatorname{Sym}(A)}=$ Aut Inv $G$ is the permutation group generated by $G \subseteq \operatorname{Sym}(A)$. In particular, every permutation group $G \leq \operatorname{Sym}(A)$ can be represented as $G=$ Aut $Q$ for a suitable set $Q$ of relations. $G$ is called $m$-closed if $Q \subseteq \operatorname{Rel}^{(m)}(A)$.

On the relational side the Galois closures are the relational and Krasner clones, respectively. A set $Q \subseteq \operatorname{Rel}(A)$ is called relational clone or Krasner clone, resp., (the latter goes back to Krasner (1938)) if every relation which can be defined by a primitive positive first order formula ( $\mathrm{ppFOF}$ ) or a first order formula (FOF), respectively, from relations in $Q$, again belongs to $Q$. We have: $[Q]_{\mathrm{RC}}=\operatorname{Inv} \operatorname{Pol} Q$ is the relational clone generated by $Q$, and $[Q]_{\mathrm{KC}}=$ Inv Aut $Q$ is the Krasner clone generated by $Q$. We mention that for finite $A$ with at least three elements we have $[Q]_{\mathrm{KC}}=[Q \cup\{\nu\}]_{\mathrm{RC}}$, where $v:=\left\{(x, y) \in A^{2} \mid x \neq y\right\}$ is the inequality relation (Pöschel and Kalužnin 1979, 1.3.5(vi)').

For more details we refer to Kerkhoff et al. (2014), Pöschel (2004) and Pöschel and Kalužnin (1979), see also Remark 3.10.

\section{The general case of an arbitrary group action}

In this section we consider a Galois connection between two-valued functions $f \in$ $\{0,1\}^{B}$ (equivalently characterizable by their support $\operatorname{supp}(f) \in \mathfrak{P}(B)$ ) and elements of a group action $\Gamma \curvearrowright B$. We shall characterize the Galois closures for this Galois connection.

Definition 3.1 Let $\Gamma \curvearrowright B$ be a group action and let $K$ be a set. A function $f \in K^{B}$ is called an invariant for a $\sigma \in \Gamma$ (and $\sigma$ is called a symmetry of $f$ ) if $\forall x \in B$ : $f\left(x^{\sigma}\right)=f(x)$. For this we use the notation $\sigma \vdash f$.

Notation 3.2 According to 2.2, the relation $\vdash$ induces a Galois connection between group elements $\sigma \in \Gamma$ and functions $f \in K^{B}$. The corresponding operators and Galois closures are denoted as follows: For $F \subseteq K^{B}$ and $G \subseteq \Gamma$ let

$$
\begin{aligned}
F^{\vdash} & :=\{\sigma \in \Gamma \mid \forall f \in F: \sigma \vdash f\}, \\
G^{\vdash} & :=\left\{f \in K^{B} \mid \forall \sigma \in G: \sigma \vdash f\right\}, \\
\bar{F} & :=\left(F^{\vdash}\right)^{\vdash}, \\
\bar{G} & :=\left(G^{\vdash}\right)^{\vdash} .
\end{aligned}
$$

Remark 3.3 One can define an induced action $\Gamma \curvearrowright K^{B}$ by $f^{\sigma}\left(x^{\sigma}\right)=f(x)$ for $f \in$ $K^{B}$. Then $\sigma \vdash f$ iff $f^{\sigma}=f$. It follows that for any $F \subseteq K^{B}, F^{\vdash}$ is a subgroup of $\Gamma$; in particular, $\bar{G} \leq \Gamma$. Moreover, if $\sigma \vdash f$ and $\pi \in \Gamma$, then

$$
\left(f^{\pi}\right)^{\left(\pi^{-1} \sigma \pi\right)}=f^{\sigma \pi}=f^{\pi},
$$

so $\pi^{-1} \sigma \pi \vdash f^{\pi}$. Therefore, if $G$ is a closed subgroup of $\Gamma$, its conjugate $\pi^{-1} G \pi$ is also closed. 
Proposition 3.4 For subgroups $G, H \leq \Gamma$ and $f \in K^{B}$ we have:

(i) $f \in G^{\vdash} \Longleftrightarrow f$ is constant on each $C \in \operatorname{Orb} G$,

(ii) $\bar{G}=\bar{H} \Longleftrightarrow G^{\vdash}=H^{\vdash} \Longleftrightarrow$ Orb $G=$ Orb $H$,

(iii) $\bar{G}$ is the largest subgroup of $\Gamma$ with the same orbits (on $B$ ) as $G$,

(iv) $\bar{G}=\left\{\sigma \in \Gamma \mid \forall b \in B: b^{\sigma} \in b^{G}\right\}$.

Proof (i) follows from the definitions and the fact that two elements $c, c^{\prime} \in B$ belong to the same orbit $C \in \operatorname{Orb}(G)$ if and only if $\exists \sigma \in G: c^{\prime}=c^{\sigma}$. Concerning (ii), the first equivalence is true for every Galois connection, the second immediately follows from (i). Condition (iii) follows from (ii) and $G \subseteq \bar{G}$ and, finally, (iv) easily follows from (iii).

The following theorem partially describes the inner structure of Galois closed groups as special subgroups of direct products of full symmetric groups. For $B^{\prime} \subseteq B$, here $\operatorname{Sym}_{B}\left(B^{\prime}\right)$ denotes the image of the natural embedding $\sigma \mapsto \sigma_{B}$ of $\operatorname{Sym}\left(B^{\prime}\right)$ into $\operatorname{Sym}(B)$ where, for $b \in B$,

$$
b^{\sigma_{B}}:= \begin{cases}b^{\sigma} & \text { if } b \in B^{\prime} \\ b & \text { otherwise }\end{cases}
$$

In the following we also use the product of subsets of a group as the set of all products of its elements, e.g., $\operatorname{Sym}_{B}\left(B_{1}\right) \cdot \operatorname{Sym}_{B}\left(B_{2}\right):=\left\{\sigma_{1} \sigma_{2} \mid\left(\sigma_{1}, \sigma_{2}\right) \in \operatorname{Sym}_{B}\left(B_{1}\right) \times\right.$ $\left.\operatorname{Sym}_{B}\left(B_{2}\right)\right\}$. If $B_{1}$ and $B_{2}$ are disjoint, then this is a direct product and thus again a subgroup of $\operatorname{Sym}(B)$. For more than two factors we use the abbreviation

$$
\operatorname{Sym}_{B}\left(B_{1}, \ldots, B_{r}\right):=\operatorname{Sym}_{B}\left(B_{1}\right) \cdot \ldots \cdot \operatorname{Sym}_{B}\left(B_{r}\right)
$$

Theorem 3.5 Let $\Gamma \curvearrowright_{h} B$ be an action of a group $\Gamma$ on $B$. The Galois closed subgroups $G$ of $\Gamma$ are exactly those of the form

$$
G=\Gamma_{B_{1}} \cap \ldots \cap \Gamma_{B_{r}}=h^{-1}\left(\operatorname{Sym}_{B}\left(B_{1}, \ldots, B_{r}\right) \cap \widehat{\Gamma}\right),
$$

where $\left\{B_{1}, \ldots, B_{r}\right\}$ is an arbitrary partition of $B$. Moreover, if $G \leq \Gamma$ and $\operatorname{Orb}(G)=$ $\left\{B_{1}, \ldots, B_{r}\right\}$, then

$$
\bar{G}=h^{-1}\left(\operatorname{Sym}_{B}\left(B_{1}, \ldots, B_{r}\right) \cap \widehat{\Gamma}\right) .
$$

If $\Gamma \curvearrowright B$ is a faithful action then we have:

$$
\bar{G} \cong \operatorname{Sym}_{B}\left(B_{1}, \ldots, B_{r}\right) \cap \widehat{\Gamma} .
$$

Proof Note that $\operatorname{Sym}_{B}\left(B_{1}, \ldots, B_{r}\right)=\operatorname{Sym}_{B}\left(B_{1}\right) \cdot \ldots \cdot \operatorname{Sym}_{B}\left(B_{r}\right)$ is the set of all permutations $\widehat{\sigma} \in \operatorname{Sym}(B)$ which stabilize every $B_{i}$. Consequently we have $\sigma \in$ $h^{-1}(\widehat{\sigma}) \subseteq \Gamma_{B_{1}} \cap \ldots \cap \Gamma_{B_{r}}$ if $\widehat{\sigma} \in \widehat{\Gamma}$. Thus the second equality of (3.5.1) follows. 
Moreover, the Eq. (3.5.2) states $\bar{G}=\Gamma_{B_{1}} \cap \ldots \cap \Gamma_{B_{r}}$, the latter being the set of all elements which stabilize (setwise) the orbits of $G$, i.e., it is the largest subgroup with this property. Thus (3.5.2) follows from 3.4 (iii).

Now, (3.5.2) implies that every closed subgroup is of the form (3.5.1). It remains to show that every group of the form (3.5.1) is really Galois closed. Let $G=\Gamma_{B_{1}} \cap \ldots \cap$ $\Gamma_{B_{r}}$. Then $\left\{B_{1}^{\prime}, \ldots, B_{s}^{\prime}\right\}:=$ Orb $G$ must be a refinement of the partition $\left\{B_{1}, \ldots, B_{r}\right\}$. With (3.5.2) we get $\bar{G}=\Gamma_{B_{1}^{\prime}} \cap \ldots \cap \Gamma_{B_{s}^{\prime}} \subseteq \Gamma_{B_{1}} \cap \ldots \cap \Gamma_{B_{r}}=G \subseteq \bar{G}$. Consequently, $G=\bar{G}$ is Galois closed.

Finally, (3.5.3) trivially follows from (3.5.2), since $\Gamma \cong h(\Gamma)=\widehat{\Gamma}$ for any faithful action $\Gamma \curvearrowright_{h} B$.

The next theorem characterizes the Galois closed subgroups of $\Gamma$ via stabilizers. It generalizes the corresponding result for special actions (communicated by $\mathrm{K}$. KEARNES and E. HORVÁTH for the action $S_{n} \curvearrowright 2^{n}$; for the action $\operatorname{GL}(n, 2) \curvearrowright 2^{n}$ see Xiao (2005), Theorem B).

Theorem 3.6 Let $G \leq \Gamma$. Then we have:

$$
\bar{G}=\bigcap_{b \in B} \Gamma_{b} \cdot G .
$$

\section{Proof}

$$
\begin{aligned}
\bar{G} \underset{\text { 3.4(iv) }}{=} & \left\{\sigma \in \Gamma \mid \forall b \in B: b^{\sigma} \in b^{G}\right\} \\
& =\left\{\sigma \in \Gamma \mid \forall b \in B \exists \pi \in G: b^{\sigma}=b^{\pi}\right\} \\
& =\left\{\sigma \in \Gamma \mid \forall b \in B \exists \pi \in G: \sigma \pi^{-1} \in \Gamma_{b}\right\} \\
& =\left\{\sigma \in \Gamma \mid \forall b \in B: \sigma \in \Gamma_{b} \cdot G\right\} \\
& =\bigcap_{b \in B} \Gamma_{b} \cdot G .
\end{aligned}
$$

Now we consider the other side of the Galois connection, namely the functions in $K^{B}$. At first we shall see that the choice of $K$ has no influence to the Galois closures $\bar{G}$ of subgroups $G \leq \Gamma$ (one-element sets $K$ will be excluded). The above closure $\bar{G}=\left(G^{\vdash}\right)^{\vdash}$ (using functions $f \in K^{B}$ ) only in the next proposition will be denoted by $\bar{G}^{K}$. Then we have:

Proposition 3.7 Let $\Gamma \curvearrowright B$ be a group action and $K$ be an arbitrary set with at least two elements. Then $\bar{G}^{K}=\bar{G}^{\{0,1\}}$ for any $G \subseteq \Gamma$.

Proof For $k \in K$ and $f \in K^{B}$ define $f_{k} \in\{0,1\}^{B}$ by $f_{k}(x)=1: \Longleftrightarrow f(x)=k$. Then, for $\sigma \in \Gamma$, we have $\sigma \vdash f \Longleftrightarrow \forall k \in K: \sigma \vdash f_{k}$. It is straightforward to see that this implies $\bar{G}^{K}=\bar{G}^{\{0,1\}}$.

Therefore, from now on, we shall restrict to the case $K:=\{0,1\}$. 
Some facts 3.8 There is a canonical bijection $\chi_{B}: \mathfrak{P}(B) \rightarrow\{0,1\}^{B}: U \mapsto \chi_{B}(U)$ between the power set $\mathfrak{P}(B)$ and $\{0,1\}^{B}$, established by taking the characteristic function $\chi_{B}(U)$ for $U \subseteq B\left(\chi_{B}(U)(x)=1: \Longleftrightarrow x \in U\right)$. In the following we write simply $\chi$ instead of $\chi_{B}$. The inverse of $\chi$ is the mapping supp : $\{0,1\}^{B} \rightarrow \mathfrak{P}(B)$ : $f \mapsto \operatorname{supp}(f)$ where $\operatorname{supp}(f)$ is the support of $f$ defined by $\operatorname{supp}(f):=\{x \in B \mid$ $f(x)=1\}$. For sets $\mathfrak{U} \subseteq \mathfrak{P}(B)$ and $F \subseteq 2^{B}$ these mappings are defined elementwise: $\chi(\mathfrak{U}):=\{\chi(U) \mid U \in \mathfrak{U}\}$ and $\operatorname{supp}(F):=\{\operatorname{supp}(f) \mid f \in F\}$. Then we have for $\sigma \in \Gamma$ and $f \in 2^{B}$ (for notation see $(3.1,2.3,2.1)$ ):

$$
\sigma \vdash f \Longleftrightarrow \operatorname{supp}(f)^{\sigma}=\operatorname{supp}(f) \Longleftrightarrow \widehat{\sigma} \triangleright \operatorname{supp}(f) .
$$

Let $F \subseteq 2^{B}$ and $\mathfrak{U} \subseteq \mathfrak{P}(B)$ with $\operatorname{supp}(F)=\mathfrak{U}$ (hence $F=\chi(\mathfrak{U})$ ), let $G \subseteq \Gamma$ and define $\overline{\overline{\mathfrak{U}}}:=\operatorname{supp}(\bar{F})$ (thus $\bar{F}=\chi(\overline{\mathfrak{U}})$ ). Then (3.8.1) implies

$$
\begin{aligned}
F^{\vdash}=\left\{\sigma \in \Gamma \mid \widehat{\sigma} \in \operatorname{Aut}_{B} \mathfrak{U}\right\}, \widehat{F^{\vdash}}=\widehat{\Gamma} \cap \operatorname{Aut}_{B} \mathfrak{U}, \operatorname{supp}\left(G^{\vdash}\right)=\operatorname{Inv}_{B}^{(1)} \widehat{G}, \\
\text { consequently, } \overline{\mathfrak{U}}=\operatorname{supp}\left(F^{\vdash \vdash}\right)=\operatorname{Inv}_{B}^{(1)} \widehat{F^{\vdash}}=\operatorname{Inv}_{B}^{(1)}\left(\widehat{\Gamma} \cap \operatorname{Aut}_{B} \mathfrak{U}\right), \\
\widehat{\bar{G}}=\widehat{G^{\vdash \vdash}}=\widehat{\Gamma} \cap \operatorname{Aut}_{B} \operatorname{supp}\left(G^{\vdash}\right)=\widehat{\Gamma} \cap \operatorname{Aut}_{B} \operatorname{Inv}_{B}^{(1)} \widehat{G}=\widehat{G} .
\end{aligned}
$$

Therefore, instead of the Galois connection introduced in 3.2 induced by $\vdash$ between $\Gamma$ and $2^{B}=\{0,1\}^{B}$, equivalently we can consider the Galois connection between $\widehat{\Gamma}$ and $\mathfrak{P}(B)=\operatorname{Rel}^{(1)}(B)$ induced by $\triangleright \subseteq \operatorname{Sym}(B) \times \operatorname{Rel}(B)$ and the corresponding operators are for $\widehat{G} \subseteq \widehat{\Gamma}$ and $\mathfrak{U} \subseteq \mathfrak{P}(B)$ :

$$
\begin{aligned}
\widehat{\Gamma} \cap \operatorname{Aut}_{B} \mathfrak{U} & =\{\widehat{\sigma} \in \widehat{\Gamma} \mid \forall U \in \mathfrak{U}: \widehat{\sigma} \triangleright U\}, \\
\operatorname{Inv}_{B}^{(1)} \widehat{G} & =\{U \subseteq B \mid \forall \widehat{\sigma} \in \widehat{G}: \widehat{\sigma} \triangleright U\} .
\end{aligned}
$$

According to 2.3, $\widehat{\sigma} \triangleright U$ means $b^{\widehat{\sigma}} \in U$ for every $b \in U$ (note $U \subseteq B$ ). Note that Inv $_{B}^{(1)} \widehat{G}$ is completely determined by the orbits of $\widehat{G}$ on $B$ since each unary invariant relation is a union of orbits. Let $\operatorname{Orb}_{B} G=\left\{B_{1}, \ldots, B_{r}\right\}$ (this coincides with $\operatorname{Orb}_{B} \widehat{G}$ ). Then

$$
\operatorname{Inv}_{B}^{(1)} \widehat{G}=\left\{\bigcup_{i \in I} B_{i} \mid I \subseteq[r]\right\}
$$

Recall that for a group action $\Gamma \curvearrowright B$ we have the corresponding permutation representation $\widehat{\Gamma} \leq \operatorname{Sym}(B)$ (cf. 2.1). According to 2.3 there is always some set $Q \subseteq \operatorname{Rel}(B)$ of relations on $B$ such that $\widehat{\Gamma}=$ Aut $Q$.

Theorem 3.9 Let $\Gamma \curvearrowright B$ be a group action and $\widehat{\Gamma}=$ Aut $Q$ for some $Q \subseteq \operatorname{Rel}(B)$. For $\mathfrak{U} \subseteq \mathfrak{P}(B)$, the Galois closure is given by $\overline{\mathfrak{U}}=[Q \cup \mathfrak{U}]_{K C}^{(1)}$. For $F \subseteq 2^{B}$ we have $\bar{F}=\chi(\overline{\mathfrak{U}})$ where $\mathfrak{U}=\operatorname{supp}(F)$.

Proof According to 3.8 and 2.3 we get $\overline{\mathfrak{U}} \underset{(3.8 .3)}{=} \operatorname{Inv}^{(1)}(\widehat{\Gamma} \cap$ Aut $\mathfrak{U})=\operatorname{Inv}^{(1)}($ Aut $Q \cap$ Aut $\mathfrak{U})=\operatorname{Inv}^{(1)} \operatorname{Aut}(Q \cup \mathfrak{U}) \underset{(2.3)}{=}[Q \cup \mathfrak{U}]_{\mathrm{KC}}^{[1]}$. 
Remark 3.10 Theorem 3.9 provides a constructive characterization of the Galois closure. To see this we describe the Krasner clone closure [...] $]_{K C}$ in more detail. Let $\varphi\left(\underline{\varrho}_{1}, \ldots, \underline{\varrho}_{t} ; x\right)$ be a first-order formula with one free variable $x\left(\varphi \in \mathrm{FOF}_{1}\right)$ and with $m_{i}$-ary relation symbols (equivalently, predicate symbols) $\varrho_{i}, i \in\{1, \ldots, t\}$ and let $\varrho_{i} \subseteq B^{m_{i}}(i \in\{1, \ldots, t\})$ be concrete relations on a base set $B$ ( $\varrho_{i}$ being the interpretation of $\underline{\varrho}_{i}$ on $B$ ). Then we define the unary relation $U_{\varphi}:=\{a \in B \mid \models$ $\left.\varphi\left(\varrho_{1}, \ldots, \varrho_{t} ; a\right)\right\} \subseteq B$. For example, with unary $\varrho_{1}$, binary $\varrho_{2}, \varrho_{1} \subseteq B, \varrho_{2} \subseteq B^{2}$ and $\varphi\left(\underline{\varrho}_{1}, \underline{\varrho}_{2} ; x\right): \equiv \exists y: \underline{\varrho}_{1}(x) \wedge \underline{\varrho}_{2}(x, y)$ we get $U_{\varphi}=\{a \in B \mid \exists b \in B: a \in$ $\left.\varrho_{1} \wedge(a, b) \in \varrho_{2}\right\}$.

With this notation we have $[Q \cup \mathfrak{U}]_{\mathrm{KC}}^{(1)}=\left\{U_{\varphi} \mid \varphi \in \mathrm{FOF}_{1}(\mathfrak{U} \cup Q)\right\}$, where $\mathrm{FOF}_{1}(\mathfrak{U} \cup Q)$ denotes the set of first order formulas with one free variable and predicate symbols from the set $\{\underline{\varrho} \mid \varrho \in \mathfrak{U} \cup Q\}$, the symbol $\underline{\varrho}$ being $m$-ary whenever $\varrho \in Q \cup \mathfrak{U}$ is an $m$-ary relation.

If one allows the inequality relation $v$ (cf. 2.3), then one can restrict to primitive positive first-order formulas with one free variable $\left(\mathrm{ppFOF}_{1}\right)$, which are much easier to handle (they contain only $\exists, \wedge,=$ and variable and relation symbols). Then we have for the Galois closures:

$$
\overline{\mathfrak{U}}=[Q \cup \mathfrak{U}]_{\mathrm{KC}}^{(1)}=[Q \cup \mathfrak{U} \cup\{v\}]_{\mathrm{RC}}^{(1)}=\left\{U_{\varphi} \mid \varphi \in \operatorname{ppFOF}_{1}(Q \cup \mathfrak{U} \cup\{v\})\right\} .
$$

\section{The natural action of $S_{n}$ on [n]}

Let $S_{n}:=\operatorname{Sym}([n])$ denote the full symmetric group on $[n]:=\{1, \ldots, n\}$. In this section we consider the natural (faithful) action $\Gamma \curvearrowright_{\text {id }}[n]$ of $\Gamma:=S_{n}$ on $B:=[n]$ where $a^{\sigma}$ is the image of $a$ under the permutation $\sigma \in S_{n}$. Thus $\widehat{\Gamma}=\Gamma=S_{n}$.

As shown in Remark 3.8, the original Galois connection (induced by $\vdash$ ) equivalently can be described as the Galois connection between $\widehat{\Gamma}=S_{n}$ and $\mathfrak{P}(B)=\mathfrak{P}([n])$ (see $(3.8 .5,3.8 .6))$ which coincides here with the "classical" Galois connection Aut - Inv ${ }^{(1)}$

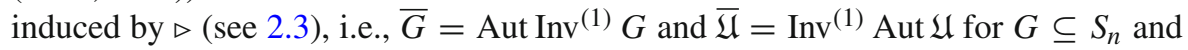
$\mathfrak{U} \subseteq \mathfrak{P}([n])$.

According to Theorem 3.5, the Galois closed subgroups are those of the form

$$
G=\operatorname{Sym}_{B}\left(B_{1}\right) \cdot \ldots \cdot \operatorname{Sym}_{B}\left(B_{r}\right)
$$

for partitions $\left\{B_{1}, \ldots, B_{r}\right\}$ of $B$. Moreover, if $G \leq \operatorname{Sym}(B)$ is any permutation group with orbits $\operatorname{Orb}(G)=\left\{B_{1}, \ldots, B_{r}\right\}$, then its closure is

$$
\bar{G}=\operatorname{Sym}_{B}\left(B_{1}\right) \cdot \ldots \cdot \operatorname{Sym}_{B}\left(B_{r}\right)
$$

If we turn to the other side of the Galois connection, we have the Galois closure $\overline{\mathfrak{U}}=\operatorname{Inv}^{(1)}$ Aut $\mathfrak{U}=[\mathfrak{U}]_{\mathrm{KC}}^{[1]}$ (this follows from Theorem 3.9 with $Q=\varnothing$, or directly from the characterization of the closure operator Inv Aut mentioned in 2.3). It is known that the unary parts of Krasner clones (and therefore the Galois closures for our Galois connection Aut $-\operatorname{Inv}^{(1)}$ ) are just sets $\mathfrak{U} \subseteq \mathfrak{P}(B)$ which are closed under 
unions, intersections and complements. This holds if and only if there is a partition $\Pi=\left\{B_{1}, \ldots, B_{r}\right\}$ of $B$ such that $\mathfrak{U}$ consists of all unions of subsets of $\Pi$ :

$$
\mathfrak{U}=\left\{\bigcup_{i \in I} B_{i} \mid I \subseteq[r]\right\} .
$$

Given any set $\mathfrak{U} \subseteq \mathfrak{P}(B)$ its closure is given by the coarsest partition $\Pi$ of $B$ such that any element of $\mathfrak{U}$ is a union of classes of $\Pi$. Clearly, this is the partition of $B$ into the orbits of $\mathrm{Aut}_{B} \mathfrak{U}$.

Remark: By the above characterization the number of closed subgroups of $S_{n}$ is equal to the Bell number $B_{n}$ of partitions of the set $[n]$. Two closed subgroups are conjugate in $S_{n}$ iff their partitions have the same distribution of class sizes, hence the number of conjugacy classes of closed subgroups is equal to the number of partitions of the integer $n$, i.e., the number of its representations as a sum of a multiset of positive integers.

\section{The action of $S_{n}$ on $2^{n}$ or $\mathfrak{P}([n])$}

As in the previous section the full symmetric group on $[n]:=\{1, \ldots, n\}$ will be denoted by $S_{n}$. In this section we consider the case $\Gamma:=S_{n}$ and $B:=2^{n}$ (cf. 3.1). So we have to deal with functions $f \in 2^{B}$, i.e., Boolean functions $f: 2^{n} \rightarrow 2$ (note that 2 is used for the set $\{0,1\}$ ), which is (as mentioned in the introduction) the "classical" approach to invariance groups. Instead of $2^{n}$ we equivalently can take the power set of an $n$-element set, say $B:=\mathfrak{P}([n])$, because there exists a bijection via the characteristic function $\chi_{[n]}$, which now we shall denote by $\psi$ (in order to avoid confusion with $\chi_{B}$ from 3.8), i.e., we have $\psi: \mathfrak{P}([n]) \rightarrow 2^{n}: I \mapsto \psi(I)$ and its inverse $\psi^{-1}: 2^{n} \rightarrow \mathfrak{P}([n])$; namely, for $\left(a_{1} \ldots, a_{n}\right) \in 2^{n}$ and $I \in \mathfrak{P}([n])$ we have $\psi(I)=\left(a_{1} \ldots, a_{n}\right) \Longleftrightarrow I=\left\{i \in[n] \mid a_{i}=1\right\}$. Sometimes we shall apply $\psi$ and $\psi^{-1}$ also to subsets $W \subseteq \mathfrak{P}([n])$ and $C \subseteq 2^{n}$ defined elementwise, i.e., $\psi(W):=\{\psi(T) \mid T \in W\}, \psi^{-1}(C):=\left\{\psi^{-1}(c) \mid c \in C\right\}$. As action of $\Gamma$ we consider the induced action $S_{n} \curvearrowright 2^{n}$ or $S_{n} \curvearrowright \mathfrak{P}([n])$ given by

$$
\left(a_{1}, \ldots, a_{n}\right)^{\widehat{\sigma}}:=\left(a_{1^{\sigma}}, \ldots, a_{n^{\sigma}}\right) \text { or } I^{\widehat{\sigma}}:=\left\{i^{\sigma} \mid i \in I\right\} \text { for } \sigma \in S_{n} \text {. }
$$

This action $S_{n} \rightarrow \operatorname{Sym}(B): \sigma \mapsto \widehat{\sigma}$ is faithful, and we use the notation from 2.1 for the image $\widehat{S}_{n}=\left\{\widehat{\sigma} \mid \sigma \in S_{n}\right\}$ which is a subgroup of $\operatorname{Sym}(B)$ with $B=2^{n}$ or $B=\mathfrak{P}([n])$, respectively, and isomorphic to $S_{n}$. From now on we shall restrict to $B:=\mathfrak{P}([n])$. As shown in 3.8, instead of the original Galois connection (induced by $\vdash$ ) we can consider the Galois connection between $\widehat{S}_{n}$ and $\mathfrak{P}(B)=\mathfrak{P}(\mathfrak{P}([n]))=\operatorname{Rel}^{(1)}(B)$ (induced by $\triangleright \subseteq \operatorname{Sym}(B) \times \operatorname{Rel}(B))$. According to (3.8.5) and (3.8.6), the corresponding operators are

$$
\begin{aligned}
\widehat{S}_{n} \cap \operatorname{Aut}_{B} \mathfrak{U} & =\left\{\widehat{\sigma} \in \widehat{S}_{n} \mid \forall U \in \mathfrak{U}: \widehat{\sigma} \triangleright U\right\} \text { for } \mathfrak{U} \subseteq \mathfrak{P}(B), \\
\operatorname{Inv}_{B}^{(1)} \widehat{G} & =\{U \subseteq B \mid \forall \widehat{\sigma} \in \widehat{G}: \widehat{\sigma} \triangleright U\} \text { for } \widehat{G} \subseteq \widehat{S}_{n} .
\end{aligned}
$$


Here $\widehat{\sigma} \triangleright U$ means $M^{\widehat{\sigma}} \in U$ for every $M \in U$ (note $\left.U \subseteq B=\mathfrak{P}([n])\right)$. In particular we have for $G \subseteq S_{n}$ (cf. (3.8.4)):

$$
\widehat{\bar{G}}=\widehat{\widehat{G}}=\widehat{S}_{n} \cap \operatorname{Aut}_{B} \operatorname{Inv}_{B}^{(1)} \widehat{G} .
$$

In order to apply Theorem 3.9 we have to characterize $\widehat{S}_{n}$ as Aut $Q$ for some set $Q \subseteq \operatorname{Rel}(B)$ of relations on $B=\mathfrak{P}([n])$. The following proposition shows that $\widehat{S}_{n}$ is 2-closed, i.e., that at most binary relations suffice. Consider the unary relations $\Theta_{i}:=\mathfrak{P}_{i}([n]):=\{M \in \mathfrak{P}([n])|| M \mid=i\} \subseteq B$ for $i \in\{0,1, \ldots, n\}$, and the binary relation (inclusion) $\iota:=\left\{\left(M, M^{\prime}\right) \mid M \subseteq M^{\prime} \subseteq[n]\right\} \subseteq B^{2}$. Then we have:

Proposition 5.1 $\widehat{S}_{n}=\operatorname{Aut}_{B}\left\{\iota, \Theta_{0}, \Theta_{1}, \ldots, \Theta_{n}\right\}$.

Proof Obviously, $\widehat{S}_{n}$ preserves $\iota$ as well as all $\Theta_{i}(i \in\{1, \ldots, n\})$, therefore $\widehat{S}_{n} \subseteq$ Aut $\left\{\iota, \Theta_{1}, \ldots, \Theta_{n}\right\}$.

Conversely, let $\pi \in \operatorname{Aut}\left\{\iota, \Theta_{1}, \ldots, \Theta_{n}\right\}$. Define $\sigma \in S_{n}$ via $\left\{a^{\sigma}\right\}:=\{a\}^{\pi}$ for $a \in[n]$ (note that the right side must be a 1-element set since $\pi \triangleright \Theta_{1}$ ). We are going to show $\pi=\widehat{\sigma}$ (thus $\pi \in \widehat{S}_{n}$ and we are done). In fact, $a \in M \subseteq[n]$ implies $(\{a\}, M) \in \iota$, thus $\left(\{a\}^{\pi}, M^{\pi}\right) \in \iota$, i.e., $\{a\}^{\pi} \subseteq M^{\pi}$ and we conclude $a^{\sigma} \in M^{\pi}$. Consequently, $M^{\pi} \supseteq\left\{a^{\sigma} \mid a \in M\right\}=M^{\widehat{\sigma}}$. But since $\pi \triangleright \Theta_{i}$ for $i:=|M|$, we have $\left|M^{\pi}\right|=|M|$ and therefore $M^{\pi}=M^{\widehat{\sigma}}$ because $\left|M^{\widehat{\sigma}}\right|=|M|$.

From 3.9 and 5.1 now follows:

Theorem 5.2 Consider the action $S_{n} \curvearrowright B$ with $B=\mathfrak{P}([n])$. Then the Galois closure $\overline{\mathfrak{U}}=\operatorname{Inv}_{B}^{(1)}\left(\widehat{S}_{n} \cap \operatorname{Aut}_{B} \mathfrak{U}\right)$ of $\mathfrak{U} \subseteq \mathfrak{P}(B)$ is given by $\overline{\mathfrak{U}}=\left[\left\{\iota, \Theta_{1}, \ldots, \Theta_{n}\right\} \cup \mathfrak{U}\right]_{K C}^{(1)}$.

The other side of the Galois connection concerns the invariance groups, i.e., the characterization of the Galois closure $\bar{G}$ for $G \subseteq S_{n}$. Applying Theorem 3.6 we get: $\bar{G}=\bigcap_{M \in \mathfrak{P}([n])}\left(S_{n}\right)_{M} \cdot G$ (here $\left(S_{n}\right)_{M}$ denotes the set stabilizer of $M \subseteq[n]$ for the natural action $S_{n} \curvearrowright[n]$, which coincides with the point stabilizer of the "point" $M \in B=\mathfrak{P}([n])$ for the action $\left.S_{n} \curvearrowright \mathfrak{P}([n])\right)$. With the above described Galois connection (cf. (5.0.3)), i.e. in terms of permutations in $\widehat{S}_{n}$, equivalently we get $\widehat{\bar{G}}=$ $\widehat{\widehat{G}}=\bigcap_{M \in B}\left(\widehat{S}_{n}\right)_{M} \cdot \widehat{G}$ (where $\left(\widehat{S}_{n}\right)_{M}$ is the stabilizer of the element $M \in B=\mathfrak{P}([n])$, cf. 2.1). From the definitions it becomes clear that the stabilizers $\left(S_{n}\right)_{M} \cong\left(\widehat{S}_{n}\right)_{M}$ are isomorphic to the direct product $\operatorname{Sym}(M) \times \operatorname{Sym}([n] \backslash M)$.

Quite another approach gives a new view to the invariance groups. Every permutation group $G \leq S_{n}$ can be presented as $G=$ Aut $Q$ for some set $Q \subseteq \operatorname{Rel}([n])$ (clearly, since $G=$ Aut Inv $G$ according to 2.3, one always can take $Q=\operatorname{Inv} G$, but this might be not the optimal choice). Therefore it is natural to ask, how one can characterize the invariance groups via their invariant relations. The answer is given in the next theorem: the invariance groups are exactly those permutation groups which can be defined with irreflexive, symmetric relations. We need some notation. Let $\operatorname{Rel}_{\text {irr sym }}^{(m)}(A)$ denote set of all $m$-ary irreflexive (i.e., $\left(a_{1}, \ldots, a_{m}\right) \in \varrho$ implies $\mid\left\{\left(a_{1}, \ldots, a_{m}\right\} \mid=m\right)$ and symmetric (i.e., $\left(a_{1}, \ldots, a_{m}\right) \in \varrho$ and $\sigma \in S_{m}$ implies $\left(a_{1^{\sigma}}, \ldots, a_{m^{\sigma}}\right) \in \varrho$ ) relations $\varrho$ on a set $A$ and let $\operatorname{Rel}_{\text {irr sym }}(A):=\bigcup_{m \in \mathbb{N}_{+}} \operatorname{Rel}_{\text {irr sym }}^{(m)}(A)$. Further, for $G \subseteq S_{n}$, we put $\operatorname{Inv}_{\text {irr sym }}^{(m)} G:=\operatorname{Rel}_{\text {irr sym }}^{(m)}([n]) \cap \operatorname{Inv}^{(m)} G$ and $\operatorname{Inv}_{\text {irr sym }} G:=\operatorname{Rel}_{\text {irr sym }}([n]) \cap \operatorname{Inv} G$. 
Theorem 5.3 For $G \subseteq S_{n}$ we have $\bar{G}=$ Aut Invirr sym $_{\text {ing }}$.

Proof Recall $B=\mathfrak{P}([n])$. The crucial point of the proof is that we can establish a bijection between subsets $U \subseteq \Theta_{m}=\mathfrak{P}_{m}([n]) \subseteq B$ and $m$-ary irreflexive symmetric relations $\varrho \in \operatorname{Rel}_{\text {irr sym }}^{(m)}([n])$ (for $m \in\{1, \ldots, n\}$ ) such that

$$
\sigma \triangleright \varrho \Longleftrightarrow \widehat{\sigma} \triangleright U \text { for } \sigma \in S_{n} \text {, consequently }\left(\widehat{\operatorname{Aut}_{[n]}} \varrho\right)=\operatorname{Aut}_{B} U \text {. }
$$

In fact, the bijection $U \mapsto \varrho$ is given by $\left(a_{1}, \ldots, a_{m}\right) \in \varrho \Longleftrightarrow\left\{a_{1}, \ldots, a_{m}\right\} \in U$. The property (5.3.1) is easy to check.

Next we mention that each $U \subseteq B$ is the union of the subsets $U_{m}:=U \cap \Theta_{m}$ for $m \in$ $\{1, \ldots, n\}$. Since $\widehat{S}_{n}$ preserves each $\Theta_{m}$ we get $\widehat{S}_{n} \cap$ Aut $_{B} U=\widehat{S}_{n} \cap$ Aut $_{B}\left\{U_{1}, \ldots, U_{n}\right\}$ (equivalently we have $\left[\{U\} \cup\left\{\Theta_{1}, \ldots, \Theta_{n}\right\}\right]_{K C}=\left[\left\{U_{1}, \ldots, U_{n}\right\} \cup\left\{\Theta_{1}, \ldots, \Theta_{n}\right\}\right]_{\mathrm{KC}}$ ). Therefore the Galois closure $\widehat{G}=\widehat{S}_{n} \cap \operatorname{Aut}_{B} \operatorname{Inv}_{B}^{(1)} \widehat{G}$ does not change if we allow only such unary invariants $U \in \operatorname{Inv}_{B}^{(1)} \widehat{G}$ with $U \subseteq \Theta_{m}$ for some $m \in\{1, \ldots, n\}$.

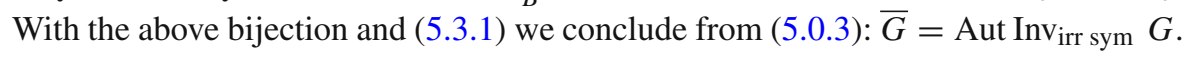

Example 5.4 Let $n=4, B=2^{4}$. We look at the Galois connection starting with Boolean functions. Consider the Boolean functions $f_{1}, f_{2}: 2^{4} \rightarrow 2$, given by

$$
\begin{aligned}
& f_{1}\left(x_{1}, x_{2}, x_{3}, x_{4}\right)=x_{1} x_{2} x_{3} x_{4} \vee x_{1} x_{2} x_{3}^{\prime} x_{4}^{\prime} \vee x_{1}^{\prime} x_{2}^{\prime} x_{3} x_{4} \\
& f_{2}\left(x_{1}, x_{2}, x_{3}, x_{4}\right)=x_{1} x_{2} x_{3} x_{4} \vee x_{1} x_{2}^{\prime} x_{3} x_{4}^{\prime} \vee x_{1}^{\prime} x_{2} x_{3}^{\prime} x_{4},
\end{aligned}
$$

(here $x_{1} x_{2}$ and $x^{\prime}$ denote conjuction and negation, respectively). We get the following supports:

$$
\begin{aligned}
& \operatorname{supp}\left(f_{1}\right)=\{(1,1,0,0),(0,0,1,1),(1,1,1,1)\} \subseteq B, \\
& \operatorname{supp}\left(f_{2}\right)=\{(1,0,1,0),(0,1,0,1),(1,1,1,1)\} \subseteq B .
\end{aligned}
$$

Correspondingly, with $B=\mathfrak{P}([4])$ instead of $B=2^{4}$ (and using the bijection $\psi$ : $\mathfrak{P}([4]) \rightarrow 2^{4}$ applied to subsets as introduced above), we can consider $\mathfrak{U}:=\left\{W_{1}, W_{2}\right\}$ instead of $F:=\left\{f_{1}, f_{2}\right\}$, where

$$
\begin{aligned}
& W_{1}=\psi^{-1}\left(\operatorname{supp}\left(f_{1}\right)\right)=\{\{1,2\},\{3,4\},\{1,2,3,4\}\}, \\
& W_{2}=\psi^{-1}\left(\operatorname{supp}\left(f_{2}\right)\right)=\{\{1,3\},\{2,4\},\{1,2,3,4\}\} .
\end{aligned}
$$

One can easily compute that for $F=\left\{f_{1}, f_{2}\right\}$ we have

$$
G:=F^{\vdash}=\{e,(12)(34),(13)(24),(14)(23)\} .
$$

This is just Klein's 4-group, which is elementary abelian. Note that $\widehat{G}=\widehat{S}_{4} \cap$ Aut $_{B} \mathfrak{U}$ according to (3.8.5). This group acts transitively on $\Theta_{i}$ for $i \in\{1,3,4\}$ yielding the 
trivial orbits $\Theta_{i}$. For $i=2$ we have

$$
\begin{aligned}
\operatorname{Orb}_{\Theta_{2}} G=\operatorname{Orb}_{\Theta_{2}} \widehat{G} & =\{\{\{1,2\},\{3,4\}\},\{\{1,3\},\{2,4\}\},\{\{1,4\},\{2,3\}\}\} \\
& =\left\{W_{1} \cap \Theta_{2}, W_{2} \cap \Theta_{2}, W_{3}\right\}, \\
\text { where } W_{3} & =\{\{1,4\},\{2,3\}\} .
\end{aligned}
$$

Note that $W_{3}$ corresponds to the Boolean function $f_{3}\left(x_{1}, x_{2}, x_{3}, x_{4}\right):=x_{1} x_{2}^{\prime} x_{3}^{\prime} x_{4} \vee$ $x_{1}^{\prime} x_{2} x_{3} x_{4}^{\prime}$ with $\operatorname{supp}\left(f_{3}\right)=\{(1,0,0,1),(0,1,1,0)\}=\psi\left(W_{3}\right)$. Because $W_{3}$ is an orbit, it belongs to Inv ${ }^{(1)} \widehat{G}=\overline{\mathfrak{U}}$, cf. (3.8.3). Consequently, according to Theorem 5.2 and Remark 3.10, there must exist a first order formula which witnesses $W_{3} \in\left[\left\{\iota, \Theta_{1}, \ldots, \Theta_{n}\right\} \cup \mathfrak{U}\right]_{\mathrm{KC}}^{(1)}$. We want to demonstrate this explicitly. One can immediately check

$$
W_{3}=\left\{\left\{x_{1}, x_{2}\right\} \in \mathfrak{P}([4]) \mid\left\{x_{1}, x_{2}\right\} \in \Theta_{2} \wedge \exists y:\left\{x_{1}, y\right\} \in W_{1} \wedge\left\{y, x_{2}\right\} \in W_{2}\right\} .
$$

This easily can be translated into a formula over the base set $B=\mathfrak{P}([4])$ with predicates from $\left\{\iota, \Theta_{1}, \ldots, \Theta_{n}\right\} \cup \mathfrak{U}$ (take $X_{1}=\left\{x_{1}\right\}, X_{2}=\left\{x_{2}\right\}, Y=\{y\}, Z=\left\{x_{1}, x_{2}\right\}$, $Z_{1}=\left\{x_{1}, y\right\}, Z_{2}=\left\{y, x_{2}\right\}$ and note that $\iota$ is the inclusion relation) yielding:

$$
\begin{aligned}
W_{3}=\{Z \in B \mid & Z \in \Theta_{2} \wedge \exists X_{1}, X_{2}, Y \in \Theta_{1}: X_{1} \neq X_{2}, X_{1} \neq Y, X_{2} \neq Y, \\
& \wedge \exists Z_{1}, Z_{2} \in \Theta_{2}: Z_{1} \in W_{1}, Z_{2} \in W_{2}, \\
& \left.\wedge X_{1} \subseteq Z_{1}, Y \subseteq Z_{1}, X_{2} \subseteq Z_{2}, Y \subseteq Z_{2}, X_{1} \subseteq Z, X_{2} \subseteq Z\right\}
\end{aligned}
$$

According to (3.8.7) (cf. also Sect. 4), the Galois closure $\overline{\mathfrak{U}}$ consists of all sets $U \subseteq \mathfrak{P}([4])$ which are unions of the above mentioned orbits $\Theta_{1}, W_{1} \cap \Theta_{2}, W_{2} \cap \Theta_{2}$, $W_{3}, \Theta_{3}, \Theta_{4}$.

By construction, $G=F^{\vdash}$ is Galois closed $(G=\bar{G})$. This is the only example of a closed subgroup of $S_{4}$ which is not the invariance group of a single Boolean function (cf. Kisielewicz (1998), Theorem 2.3) and thus a counterexample to Clote and Kranakis (1991), Theorem 6.

According to Theorem 5.3, $G$ is also the automorphism group of its irreflexive invariant relations. In fact we conclude from the proof of 5.3:

$$
\begin{aligned}
G & =\operatorname{Aut}_{[4]}\left\{\varrho_{1}, \varrho_{2}\right\} \text { where } \\
\varrho_{1} & =\{(1,2),(2,1),(3,4),(4,3)\}, \quad \varrho_{2}=\{(1,3),(3,1),(2,4),(4,2)\} .
\end{aligned}
$$

Example 5.5 Let $n=4, \Gamma=S_{4}$ and take the action $\Gamma \curvearrowright B$ with $B:=\mathfrak{P}([4])$. We consider the Galois connection (induced by $\vdash$, cf. 3.2) starting with the group $G:=$ $\left\langle\left(\begin{array}{ll}1234 \\ 2\end{array}\right)\right\rangle_{S_{4}}=\{e,(1234),(13)(24),(1432)\}$ and want to compute the closure $\bar{G}$ at first via Theorem 3.6. Therefore we need $\Gamma_{b} \cdot G$ for all $b \in B=\mathfrak{P}([4])$.

Let

$$
\begin{aligned}
H & :=\langle(1234),(13)\rangle_{S_{4}} \\
& =\{e,(1234),(13)(24),(1432),(13),(14)(23),(24),(12)(34)\} .
\end{aligned}
$$


This subgroup of $S_{4}$ is the dihedral group (symmetry group of a square with points $1,2,3,4)$ and equals $G \cup(13) \cdot G$. With this we can compute:

$$
\begin{aligned}
\Gamma_{\{1,3\}} \cdot G= & \Gamma_{\{2,4\}} \cdot G=H, \\
\Gamma_{\{1,2\}} \cdot G= & \Gamma_{\{3,4\}} \cdot G=H \cup(34) \cdot H \\
= & \{e,(1234),(13)(24),(1432),(13),(14)(23),(24),(12)(34)\} \\
& \cup\{(34),(123),(1324),(142),(134),(1423),(243),(12)\}, \\
\Gamma_{\{1,4\}} \cdot G= & \Gamma_{\{2,3\}} \cdot G=H \cup(23) \cdot H \\
= & \{e,(1234),(13)(24),(1432),(13),(14)(23),(24),(12)(34)\} \\
& \cup\{(23),(124),(1342),(143),(132),(14),(234),(1243)\}, \\
\Gamma_{b} \cdot G= & S_{4} \text { for all other } b \in \mathfrak{P}([4]) .
\end{aligned}
$$

Consequently, $\bar{G}=\bigcap_{b \in B} \Gamma_{b} \cdot G=H$ is the Galois closure of $G$.

We also want to demonstrate the other approach to the closure $\bar{G}$ via Theorem 5.3. According to the bijection mentioned in the proof of 5.3, we can represent an $m$-ary irreflexive symmetric relation $\varrho$ by its corresponding subset $U$ of $\Theta_{m} \subseteq \mathfrak{P}([n])$ (the set of all $m$-element subsets of $[n]$ ). Every $\sigma \in G$ must stabilize each such $U$ (cf. 5.3.1). It is enough to consider the orbits of the action $G \curvearrowright \mathfrak{P}([n])$ (the other invariants are unions of orbits). These orbits can be computed easily:

$$
\begin{array}{ll}
B_{0}:=\emptyset^{G} & =\{\emptyset\}, \\
B_{1}:=\{1\}^{G} & =\{\{1\},\{2\},\{3\},\{4\}\}, \\
B_{2}:=\{1,2\}^{G} & =\{\{1,2\},\{1,4\},\{2,3\},\{3,4\}\}, \\
B_{3}:=\{1,3\}^{G}=\{\{1,3\},\{2,4\}\}, \\
B_{4}:=\{1,2,3\}^{G}=\{\{1,2,3\},\{1,2,4\},\{1,3,4\},\{2,3,4\}\}, \\
B_{5}:=\{1,2,3,4\}^{G}=\{\{1,2,3,4\}\} .
\end{array}
$$

The automorphism group of $B_{2}$ as well as of $B_{3}$ is the above dihedral group $H$ while every $\sigma \in S_{4}$ stabilizes each other orbit. Thus we also get $\bar{G}=$ $H$.

Note that the Boolean function $f_{2}$ from example 5.4 corresponds to $W_{2}=B_{3} \cup B_{5}$ $\left(\psi\left(W_{2}\right)=\operatorname{supp}\left(f_{2}\right)\right)$. Thus we can conclude $\bar{G}=\left\{f_{2}\right\}^{\vdash}$ (because Aut $W_{2}=$ Aut $\left.B_{3}=H\right)$.

Example 5.6 For small values of $n$ we can compute the Galois closed subgroups of $S_{n}$ and their conjugacy classes (i.e., the orbits of $S_{n}$ acting on the subgroups of $S_{n}$ by conjugation). The following table gives the total number of (closed) subgroups and of conjugacy classes of (closed) subgroups. 


\begin{tabular}{|r||r|r|r|r|}
\hline \multicolumn{1}{|l||}{$\begin{array}{l}\text { conjugacy classes } \\
\text { of subgroups }\end{array}$} & $\begin{array}{l}\text { conjugacy classes } \\
\text { of closed groups }\end{array}$ & \multicolumn{1}{c|}{ subgroups } & \multicolumn{1}{l|}{$\begin{array}{l}\text { closed } \\
\text { subgroups }\end{array}$} \\
\hline \hline 2 & 2 & 2 & 2 & 2 \\
\hline 3 & 4 & 3 & 6 & 5 \\
\hline 4 & 11 & 8 & 30 & 22 \\
\hline 5 & 19 & 11 & 156 & 93 \\
\hline 6 & 56 & 35 & 1,455 & 955 \\
\hline 7 & 96 & 51 & 11,300 & 6,812 \\
\hline 8 & 296 & 168 & 151,221 & 99,492 \\
\hline 9 & 554 & 254 & $1,694,723$ & $1,015,565$ \\
\hline
\end{tabular}

\section{The action of $\mathrm{GL}(n, 2)$ on $2^{n}$}

In this section we specialize the scenario $3.1,3.2$ to the case $\Gamma=\operatorname{GL}(n, 2)$, the group of invertible linear maps on the set $B:=2^{n}$, the latter considered as $n$-dimensional vector space over the 2-element field. For short, here we also use the notation $V:=2^{n}$ and $L_{n}:=\operatorname{GL}(n, 2)$.

Each element of $L_{n}$ is a linear map of the form $\sigma: V \rightarrow V: x \mapsto M x$ for some invertible $n \times n$-matrix $M$ ( $M x$ is the product of the matrix with the (column) vector $x \in V)$. We have $L_{n} \leq \operatorname{Sym}(V)$ and we take the natural action on $V$ as in Sect. 4 (here with $V$ instead of $[n]$ ), in particular we have $\widehat{L}_{n}=L_{n}$. Therefore the Galois connection to be considered (according to $(3.8 .5,3.8 .6))$ is given by

$$
\begin{aligned}
\operatorname{Lin} \mathfrak{U} & :=L_{n} \cap \operatorname{Aut}_{V} \mathfrak{U}=\left\{\sigma \in L_{n} \mid \forall U \in \mathfrak{U}: \sigma \triangleright U\right\} \text { for } \mathfrak{U} \subseteq \mathfrak{P}(V), \\
\operatorname{Inv}_{V}^{(1)} G & :=\{U \subseteq V \mid \forall \sigma \in G: \sigma \triangleright U\} \text { for } G \subseteq L_{n} .
\end{aligned}
$$

In order to apply Theorem 3.9 we need some $Q \subseteq \operatorname{Rel}(V)$ such that $L_{n}=\operatorname{Aut}_{V} Q$. We show that $L_{n}$ is 3-closed, more concretely it is determined by the graph $R:=$ $\left\{(u, v, w) \in V^{3} \mid u+v=w\right\}$ of the vector space addition + . We have:

Proposition 6.1 $L_{n}=\operatorname{Aut}_{V}\{R\}$.

Proof Let $\sigma \in$ Aut $_{V}\{R\}$. Then $\sigma \triangleright R$ implies $\sigma(x+y)=\sigma(x)+\sigma(y)$, i.e., $\sigma$ is linear. Thus $\operatorname{Aut}_{V}\{R\} \subseteq L_{n}$. The converse inclusion is trivial since each linear map preserves $R$.

Thus from 3.9 and 6.1 follows:

Theorem 6.2 Let $\mathfrak{U} \subseteq \mathfrak{P}(V)$. Then the Galois closure $\overline{\mathfrak{U}}=\operatorname{Inv}^{(1)}$ Lin $\mathfrak{U}$ is given by $\overline{\mathfrak{U}}=[\{R\} \cup \mathfrak{U}]_{K C}^{(1)}$.

Example 6.3 Let $U \leq V$ be a nontrivial subspace of $V$. Then the Galois closures generated by the singleton $\mathfrak{U}=\{U\}$ is easy to describe:

$$
\operatorname{Orb} \operatorname{Lin}\{U\}=\{\{o\}, U \backslash\{o\}, V \backslash U\},
$$


$o$ denoting the zero vector $(0, \ldots, 0)$ (note that by (3.8.7) it is enough to consider the orbits). This follows from elementary linear algebra.

If $U$ is not a subspace, then the situation is more complicated and it would be interesting to have a "nice" description for Orb $\operatorname{Lin}\{U\}$ also in this case.

Example 6.4 Let $n=4$ and $\Gamma=L_{4}$ acting on $V=2^{4}$. As in Example 5.4 we consider $F:=\left\{f_{1}, f_{2}\right\}$ with the Boolean functions

$$
\begin{aligned}
f_{1}\left(x_{1}, x_{2}, x_{3}, x_{4}\right) & =x_{1} x_{2} x_{3} x_{4} \vee x_{1} x_{2} x_{3}^{\prime} x_{4}^{\prime} \vee x_{1}^{\prime} x_{2}^{\prime} x_{3} x_{4}, \\
f_{2}\left(x_{1}, x_{2}, x_{3}, x_{4}\right) & =x_{1} x_{2} x_{3} x_{4} \vee x_{1} x_{2}^{\prime} x_{3} x_{4}^{\prime} \vee x_{1}^{\prime} x_{2} x_{3}^{\prime} x_{4}, \\
\text { with } \quad R_{1}:=\operatorname{supp}\left(f_{1}\right) & =\{(1,1,0,0),(0,0,1,1),(1,1,1,1)\}, \\
R_{2}:=\operatorname{supp}\left(f_{2}\right) & =\{(1,0,1,0),(0,1,0,1),(1,1,1,1)\} .
\end{aligned}
$$

Because $L_{4}$ is much larger than $S_{4}$ (see 6.5) we do not want to go into the details of the computation of the Galois closures $G:=F^{\vdash}$ and $\bar{F}=F^{\vdash \vdash}$. With $\mathfrak{U}:=\left\{R_{1}, R_{2}\right\}$ we have $G=\operatorname{Lin} \mathfrak{U}$. It turns out that this is a group with 32 elements. For the description of $\bar{F}$ it is enough to determine the orbits of $G$ on $V$, then their unions give every invariant from $\overline{\mathfrak{U}}=\operatorname{supp}(\bar{F})(\mathrm{cf}$. (3.8.7)). These orbits must be the minimal (w.r.t. inclusion) sets in $[\{R\} \cup \mathfrak{U}]_{\mathrm{KC}}^{(1)}$ (cf. Theorem 6.2) and they can be obtained as follows:

$$
\begin{aligned}
& O_{0}:=\{(0,0,0,0)\}=\{w \in V \mid \exists v:(v, v, w) \in R\}, \\
& O_{1}:=\{(1,1,1,1)\}=R_{1} \cap R_{2}, \\
& O_{2}:=\{(1,1,0,0),(0,0,1,1)\}=R_{1} \backslash O_{1}, \\
& O_{3}:=\{(1,0,1,0),(0,1,0,1)\}=R_{2} \backslash O_{1}, \\
& O_{4}:=\{(1,0,0,1),(0,1,1,0)\}=O_{2}+O_{3}, \\
& O_{5}:=V \backslash\left(O_{0} \cup O_{1} \cup O_{2} \cup O_{3} \cup O_{4}\right) .
\end{aligned}
$$

\begin{tabular}{|c|c|c|c|c|c|c|}
\hline$n$ & $|V|$ & $\left|L_{n}\right|$ & $\begin{array}{l}\text { conjugacy } \\
\text { classes of } \\
\text { subgroups }\end{array}$ & $\begin{array}{l}\text { conjugacy } \\
\text { classes of closed } \\
\text { subgroups }\end{array}$ & subgroups & $\begin{array}{l}\text { closed } \\
\text { subgroups }\end{array}$ \\
\hline 2 & 4 & 6 & 4 & 3 & 6 & 5 \\
\hline 3 & 8 & 168 & 15 & 9 & 179 & 100 \\
\hline 4 & 16 & 20160 & 137 & 43 & 48337 & 12537 \\
\hline
\end{tabular}

Note that the representation of each orbit in terms of $R_{1}$ and $R_{2}$ easily can be expressed by a first order formula (using the vector space addition encoded in $R$, e.g., $O_{4}=O_{2}+O_{3}:=\left\{v_{1}+v_{2} \mid v_{1} \in O_{2}, v_{2} \in O_{3}\right\}$ can be expressed as $\{w \in V \mid$ $\left.\left.\exists v_{1} \exists v_{2}: v_{1} \in O_{1} \wedge v_{2} \in O_{2} \wedge\left(v_{1}, v_{2}, w\right) \in R\right\}\right)$.

Example 6.5 For small values of $n$, the following table gives the total number of (closed) subgroups and of conjugacy classes of (closed) subgroups of $L_{n}=\operatorname{GL}(n, 2)$. 
Remark 6.6 For $\sigma \in S_{n}$, let $\widetilde{\sigma}: V \rightarrow V: x \mapsto M_{\sigma} x$ be the linear mapping given by the corresponding permutation matrix $M_{\sigma}:=\left(\delta_{i}, j\right)_{i, j \in[n]}$. This group action $S_{n} \curvearrowright V$ gives an embedding $\sigma \mapsto \widetilde{\sigma}$ of $S_{n}$ into $L_{n}: \widetilde{S}_{n}:=\left\{\widetilde{\sigma} \mid \sigma \in S_{n}\right\} \leq L_{n}$.

To fix notation, let $E_{n}:=\left\{e_{1}, \ldots, e_{n}\right\}$ be the standard basis of the vector space $V$, i.e., $e_{1}=(1,0, \ldots, 0), \ldots, e_{n}=(0, \ldots, 0,1)$. Moreover, for $I \subseteq[n]$ let $e_{I}:=$ $\sum_{i \in I} e_{i}$, for $I=\emptyset$ we put $e_{\emptyset}:=o$ (the zero vector). With the notation from Sect. 5 we have $e_{I}=\psi(I)$. Thus each $x \in V$ is of the form $e_{I}$ for some $I \subseteq[n]$.

The subgroup $\widetilde{S}_{n}$ can be characterized inside $L_{n}$ by the unary relation $E_{n} \subseteq V$ :

$$
\widetilde{S}_{n}=\operatorname{Lin}\left\{E_{n}\right\}
$$

Moreover, the action $S_{n} \curvearrowright 2^{n}$ or equivalently $S_{n} \curvearrowright \mathfrak{P}([n])$ from Sect. 5 can be interpreted as $\widetilde{S}_{n} \curvearrowright V$ within the action $L_{n} \curvearrowright V$ (for $S_{n} \curvearrowright \mathfrak{P}([n])$ via the mapping $\left.\mathfrak{P}([n]) \rightarrow V: I \mapsto e_{I}\right)$. In fact, let $U \subseteq \mathfrak{P}([n])$ and $f: 2^{n} \rightarrow 2$ be the corresponding Boolean function (i.e., $\operatorname{supp}(f)=\{\psi(I) \mid I \in U\}$ ). Then we have with the notation from Sect. 5 ( $\widehat{\sigma}$ denotes the permutation on $B=\mathfrak{P}([n])$ induced by $\sigma \in S_{n}$ ),

$$
\left.\sigma \vdash f \Longleftrightarrow \widehat{\sigma} \in \operatorname{Aut}_{B} U \text { (i.e., } \widehat{\sigma} \triangleright U\right) \Longleftrightarrow \widetilde{\sigma} \in \operatorname{Lin}\left\{e_{I} \mid I \in U\right\} \text {. }
$$

Thus, the Galois closures of the original Galois connection induced by $\vdash$ (cf. 3.2) as well as of the Galois connection $(5.0 .1,5.0 .2)$, can be characterized as special Galois closures of the above Galois connection $(6.0 .1,6.0 .2)$.

For example, for $G \subseteq S_{n}, \mathfrak{U} \subseteq \mathfrak{P}(V), \mathfrak{W} \subseteq \mathfrak{P}(\mathfrak{P}([n]))$ and $F \subseteq 2^{\left(2^{n}\right)}$ such that $\operatorname{supp}(F)=\mathfrak{U}$ and $\mathfrak{W}=\psi^{-1}(\mathfrak{U})$, we have

$$
\begin{aligned}
G^{\vdash} & =\chi_{V}\left(\operatorname{Inv}_{V}^{(1)} \widetilde{G}\right), & G^{\vdash} & =\psi\left(\operatorname{Inv}_{B}^{(1)} \widehat{G}\right), \\
\widetilde{G^{\vdash}} & =\widetilde{S}_{n} \cap{\operatorname{Lin} \operatorname{Inv}_{V}^{(1)} \widetilde{G}} & \widehat{G^{\vdash \vdash}} & =\widehat{S}_{n} \cap \operatorname{Aut}_{B} \operatorname{Inv}_{B}^{(1)} \widehat{G} \\
& =\operatorname{Lin}\left(\left\{E_{n}\right\} \cup \operatorname{Inv}_{V}^{(1)} \widetilde{G}\right), & & =\operatorname{Aut}_{B}\left(\left\{\iota, \Theta_{1}, \ldots, \Theta_{n}\right\} \cup \operatorname{Inv}_{B}^{(1)} \widehat{G}\right), \\
\widetilde{F^{\vdash}} & =\widetilde{S}_{n} \cap \operatorname{Lin}_{V} \mathfrak{U}, & \widehat{F^{\vdash}} & =\widehat{S}_{n} \cap \operatorname{Aut}_{B} \mathfrak{W},
\end{aligned}
$$

where $\widetilde{G}:=\{\widetilde{\sigma} \mid \sigma \in G\}, \widehat{G}:=\{\widehat{\sigma} \mid \sigma \in G\}$.

Acknowledgements This manuscript has a long history of about ten years. The authors thank E. Friese, K. Kearnes, E. Lehtonen, G. Makai, S. Radeleczki and T. Waldhauser for helpful discussions during this time. Moreover we thank an anonymous reviewer for valuable suggestions to improve the presentation.

Funding Information Open Access funding enabled and organized by Projekt DEAL.

\section{Compliance with ethical standards}

Conflict of interest The authors declare that they have no conflict of interest.

Open Access This article is licensed under a Creative Commons Attribution 4.0 International License, which permits use, sharing, adaptation, distribution and reproduction in any medium or format, as long as you give appropriate credit to the original author(s) and the source, provide a link to the Creative Commons licence, and indicate if changes were made. The images or other third party material in this article are included 
in the article's Creative Commons licence, unless indicated otherwise in a credit line to the material. If material is not included in the article's Creative Commons licence and your intended use is not permitted by statutory regulation or exceeds the permitted use, you will need to obtain permission directly from the copyright holder. To view a copy of this licence, visit http://creativecommons.org/licenses/by/4.0/.

\section{References}

Clote, P., Kranakis, E.: Boolean functions, invariance groups, and parallel complexity. SIAM J. Comput. 20, 553-590 (1991)

Dalla Volta, F., Siemons, J.: Permutation groups defined by unordered relations. In: Ischia Group Theory 2008, pp. 56-67. World Sci. Publ., Hackensack (2009)

Grech, M.: Regular symmetric groups of Boolean functions. Discrete Math. 310(21), 2877-2882 (2010)

Grech, M., Kisielewicz, A.: Symmetry groups of Boolean functions. Eur. J. Combin. 40, 1-10 (2014)

Horváth, E., Makay, G., Pöschel, R., Waldhauser, T.: Invariance groups of finite functions and orbit equivalence of permutation groups. Open Math. 13(1), 83-95 (2015)

Kerkhoff, S., Pöschel, R., Schneider, F.: A short introduction to clones. In: Proceedings of the Workshop on Algebra, Coalgebra and Topology (WACT 2013). Electron. Notes Theor. Comput. Sci., vol. 303, pp. 107-120. Elsevier Sci. B. V., Amsterdam (2014)

Kisielewicz, A.: Symmetry groups of Boolean functions and constructions of permutation groups. J. Algebra 199, 379-403 (1998)

Krasner, M.: Une généralisation de la notion de corps. J. Math. Pure et Appl. 17, 367-385 (1938)

McKenzie, R., McNulty, G., Taylor, W.: Algebras, Lattices, Varieties, vol. I. Wadsworth \& Brooks, Monterey (1987)

Pöschel, R.: Galois connections for operations and relations. In: Denecke, K., Erné, M., Wismath, S. (eds.) Galois Connections and Applications, Mathematics and its Applications, vol. 565, pp. 231-258. Kluwer Academic Publishers, Dordrecht (2004)

Pöschel, R., Kalužnin, L.: Funktionen- und Relationenalgebren. Deutscher Verlag der Wissenschaften, Berlin, : Birkhäuser Verlag Basel. Math. Reihe 67, 1979 (1979)

Woldar, A.: Geometric groups of second order and related combinatorial structures. J. Combin. Designs 28(4), 307-326 (2020)

Xiao, W.: Linear symmetries of Boolean functions. Discrete Appl. Math. 149, 192-199 (2005)

Publisher's Note Springer Nature remains neutral with regard to jurisdictional claims in published maps and institutional affiliations. 